\title{
Definitive radiotherapy with or without chemotherapy for clinical stage T4N0-1 non-small cell lung cancer
}

\author{
Yeon Joo Kim, MD', Si Yeol Song, MD, PhD', Seong-Yun Jeong, PhD², Sang We Kim, MD, PhD², \\ Jung-Shin Lee, MD, PhD², Su Ssan Kim, MD, PhD', Wonsik Choi, MD, PhD², Eun Kyung Choi, MD, PhD \\ 'Department of Radiation Oncology, Asan Medical Center, University of Ulsan College of Medicine, Seoul; \\ ${ }^{2}$ Institute of Innovative Science, Asan Medical Center, Seoul; \\ ${ }^{3}$ Department of Medical Oncology, Asan Medical Center, University of Ulsan College of Medicine, Seoul; \\ ${ }^{4}$ Department of Radiation Oncology, Gangneung Asan Hospital, Gangneung, Korea
}

Purpose: To determine failure patterns and survival outcomes of T4NO-1 non-small cell lung cancer (NSCLC) treated with definitive radiotherapy.

Materials and Methods: Ninety-five patients with T4N0-1 NSCLC who received definitive radiotherapy with or without chemotherapy from May 2003 to October 2014 were retrospectively reviewed. The standard radiotherapy scheme was 66 Gy in 30 fractions. The main concurrent chemotherapy regimen was $50 \mathrm{mg} / \mathrm{m}^{2}$ weekly paclitaxel combined with $20 \mathrm{mg} / \mathrm{m}^{2}$ cisplatin or AUC 2 carboplatin. The primary outcome was overall survival (OS). Secondary outcomes were failure patterns and toxicities.

Results: The median age was 64 years (range, 34 to 90 years). Eighty-eight percent of patients $(n=84)$ had an Eastern Cooperative Oncology Group performance status of $0-1$, and $42 \%(n=40)$ experienced pretreatment weight loss. Sixty percent of patients ( $n$ = 57) had no metastatic regional lymph nodes. The median radiation dose was EQD2 67.1 Gy (range, 56.9 to 83.3 Gy). Seventy-one patients (75\%) were treated with concurrent chemotherapy; of these, 13 were also administered neoadjuvant chemotherapy. At a median follow-up of 21 months (range, 1 to 102 months), 3-year OS was 44\%. The 3-year cumulative incidences of local recurrence and distant recurrence were $48.8 \%$ and $36.3 \%$, respectively. Pretreatment weight loss and combined chemotherapy were significant factors for OS. Acute esophagitis over grade 3 occurred in three patients and grade 3 chronic esophagitis occurred in one patient. There was no grade $3-4$ radiation pneumonitis.

Conclusion: Definitive radiotherapy for T4NO-1 NSCLC results in favorable survival with acceptable toxicity rates. Local recurrence is the major recurrence pattern. Intensity modulated radiotherapy and radio-sensitizing agents would be needed to improve local tumor control.

Keywords: Carcinoma, Non-small-cell lung, Radiotherapy, Definitive, T4, Local tumor control, Survival

\section{Introduction}

Before the introduction of chemotherapy, stage III non-small cell lung cancer (NSCLC) had poor survival outcomes with single local treatments such as surgery or radiotherapy (RT). Addition of induction chemotherapy to RT significantly improved survival $[1,2]$, and subsequent trials reported that concurrent chemo-radiotherapy (CRT) was better than sequential CRT

Received 7 October 2015, Revised 1 December 2015, Accepted 9 December 2015.

Correspondence: Si Yeol Song, MD, PhD, Department of Radiation Oncology, Asan Medical Center, University of Ulsan College of Medicine, 88 Olympic-ro 43-gil, Songpa-gu, Seoul 05505, Korea. Tel: +82-2-3010-4431, Fax: +82-2-3010-6950, E-mail: coocoori@gmail.com

(c) This is an Open Access article distributed under the terms of the Creative Commons Attribution Non-Commercial License (http://creativecommons.org/ licenses/by-nc/4.0/) which permits unrestricted non-commercial use, distribution, and reproduction in any medium, provided the original work is properly cited.

www.e-roj.org 
$[3,4]$. Combined with surgery, induction chemotherapy also improved survival for NSCLC patients [5]. Moreover, the addition of neoadjuvant RT to chemotherapy was suggested to improve mediastinal downstaging and the rate of complete resection [6]. As a result, management for stage III NSCLC is currently based on resectability. For resectable patients, surgery is performed with neoadjuvant CRT or adjuvant chemotherapy and RT. On the other hand, concurrent CRT is considered the standard therapy for unresectable stage III NSCLC. However, determination of resectability is difficult in "potentially resectable" subgroups such as stage IIIA, which has led to controversy in the management of stage IIIA NSCLC patients.

Stage IIIA subgroups have markedly different clinical characteristics despite having a similar prognosis, and include patients with $\mathrm{N} 2$ disease (T1-3N2M0) and patients with either extensive local invasion or ipsilateral additional tumor nodules (T4NO-1M0). It is appropriate to assume that the stage III-N2 and T4N0-1 groups would need different treatment approaches.

A previous prospective trial compared the survival outcomes of trimodality treatment (concurrent CRT induction with an RT dose of 45 Gy followed by surgical resection) and definitive CRT with a total RT dose of 61 Gy in stage IIIA-N2 NSCLC [7]. There was no significant 5-year overall survival (OS) advantage for surgery after CRT, despite improved progression free survival (PFS). In exploratory analysis, trimodality approaches showed significantly better OS than bimodality approaches if lobectomies were performed. However, these results were only for stage IIIA-N2 disease and not stage T4NO-1 disease.

To the best of our knowledge, there has only been one study to date of the treatment outcomes of stage T4NO-1 NSCLC patients treated with definitive CRT [8]. Although the results of that report showed comparable survival with surgery, some physicians still advocate surgery combined with chemotherapy as the only treatment capable of achieving long-term survival in this subgroup [9].

In the absence of phase III studies comparing surgery to RT as a local treatment, additional information about this particular subgroup with extensive local invasion and minimal nodal disease is crucial. In contrast to the single study on definitive CRT, there have been many reports on the surgical outcomes for stage T4N0-1 patients [10-12]. We here evaluated the survival outcomes of definitive RT with or without chemotherapy in stage T4NO-1 NSCLC patients. Furthermore, by evaluating failure patterns, especially local failure and toxicities, we identified additional strategies for improving patient survival.

\section{Materials and Methods}

\section{Patients}

Between May 2003 and October 2014, 102 patients with T4NO1 disease received definitive CRT at Asan Medical Center. After excluding seven patients who ended treatment with an RT dose of $50 \mathrm{~Gy}$, we retrospectively reviewed 95 patients. The first author (Kim YJ) reviewed all patients and tumor stage was determined according to the American Joint Committee on Cancer (AJCC) 7th edition TNM stage classification. Known prognostic factors such as Eastern Cooperative Oncology Group performance status (ECOG-PS) and weight loss were also analyzed. Pretreatment weight loss was defined as $5 \%$ or more weight loss over the previous 6 months.

Clinical staging work-ups included pathologic confirmation, chest computed tomography (CT), 18-fluoro-deoxyglucose positron emission tomography computed tomography $\left({ }^{18} \mathrm{FDG}\right.$ PET-CT), brain magnetic resonance imaging, and bronchoscopy. Diagnostic thoracotomy was not mandatory and the T4 status was mainly evaluated on imaging. Lymph nodes with a diameter $\geq 1 \mathrm{~cm}$ and definite FDG uptake were classified as clinically malignant. Otherwise, pathologic confirmation was performed whenever possible.

\section{Radiotherapy}

Planning CT was obtained in a supine, arms-raised position using a $2.5 \mathrm{~mm}$-slice thickness. Four-dimensional CT (4DCT) images reflecting respiratory motion were collected from January 2005 onwards.

Gross tumor volume (GTV) of the primary lung lesion delineation was performed using the lung setting $(W=1,700$, $L=-300$ ), while GTV of the hilar and mediastinal lymph nodes was delineated in a mediastinal setting $(W=600, L=$ 40). For patients with $4 \mathrm{DCT}$, the internal target volume was also contoured. Clinical target volume (CTV) was delineated for patients with a relatively small GTV by a 5-mm expansion from the GTV. For patients with primary lung lesion invading mediastinum, we also included the involved mediastinal lymph node areas to the CTV. For bulky tumors, there was no CTV. The planning target volume (PTV) margin was $7 \mathrm{~mm}$ radially and $10 \mathrm{~mm}$ longitudinally. In patients treated with induction chemotherapy, the postchemotherapy volume was defined as the GTV. Organs at risk include healthy lung tissues, the esophagus, the spinal cord, and the heart.

The standard RT scheme was 66 Gy with 2.2 Gy per fraction. After irradiating with $44 \mathrm{~Gy}$ or $52.8 \mathrm{~Gy}$, repeated CT simulation was routinely performed. We performed chest $X$-rays (CXRs) 
every week. Patients who showed dramatic volume changes were requested for earlier repeated CT simulation. Treatment verification was performed by weekly $\mathrm{kV}$ imaging guidance using set-up correction based on carina and bony anatomy.

Three-dimensional conventional RT was used as a routine policy, and intensity modulated radiotherapy (IMRT) was considered when the following normal organ constrains were difficult to meet; maximal dose $\left(D_{\max }\right)$ for spinal cord $<50 \mathrm{~Gy}$, mean lung dose (MLD) $<20 \mathrm{~Gy}$, volume of lung receiving at least $20 \mathrm{~Gy}$ (V20) <30\%, mean esophagus dose <35 Gy.

\section{Chemotherapy}

The main concurrent chemotherapy regimen was $50 \mathrm{mg} / \mathrm{m}^{2}$ weekly paclitaxel combined with $20 \mathrm{mg} / \mathrm{m}^{2}$ cisplatin or AUC 2 carboplatin. Although concurrent CRT is a standard format for definitive CRT, patients with very large tumors and a high risk of radiation-related toxicities were initially treated with induction chemotherapy for volume reduction. The standard chemotherapy regimen was 2 cycles of 1,000 mg/ $\mathrm{m}^{2}$ gemcitabine (days 1 and 8) with $70 \mathrm{mg} / \mathrm{m}^{2}$ cisplatin (day 1) in sequential schedules every 3 weeks. Patients older than 75 years old, with a poor performance status or with poor lung function were recommended for definitive RT alone.

\section{Follow-up and toxicity scoring}

During definitive CRT, all patients were interviewed weekly with a complete blood count test and CXR. Patients were routinely followed up by a radiation oncologist and/or medical oncologist with chest CT and CXR 1 month after treatment, every 3 months during the first 2 years, and every 6 months until 5 years after treatment.

Locoregional recurrence (LR) was defined as primary tumor and regional lymph node progression. We also investigated if the recurrence occurred in radiation field or not. Distant metastasis (DM) was defined as intrapulmonary recurrence outside the radiation field or in ipsilateral different lobes, the contralateral lung, malignant pleural effusion, pleural metastasis, or other distant organs. Although we used AJCC 7th edition TNM stage classification, we considered ipsilateral different lobe metastasis as DM. We accepted that the patients with ipsilateral separate lung nodule had better prognosis than those with the other M1 factors in AJCC 6th edition. However, we assumed that there must be a difference between the assessment of initial stage and recurrence. One of the aims of the present study was to evaluate the local tumor control of definitive RT. If intrapulmonary recurrence outside the radiation field had been considered as $L R$, we would fail to show the true effect of definitive RT.

Adverse events that occurred during treatment and within 3 months after CRT were defined as acute toxicities. The severity of toxicity was assessed according to the National Cancer Institute's Common Terminology Criteria for Adverse Events (CTCAE) ver. 4.03, for hematologic complications, radiation esophagitis, and pneumonitis.

\section{Statistics}

Follow-up durations were calculated from the date that treatment started. Survival was calculated using Kaplan-Meier method from the date treatment started until death. The $p$-values were determined by the log-rank test for univariate analysis and with a Cox proportional hazard model for multivariate analysis. Significant factors revealed by univariate analysis $(p<0.05)$ were included for multivariate analysis. Cumulative incidence of LR and DM were also calculated using Kaplan-Meier method (one minus survival). A Student t-test was used to assess the relationship between chemotherapy and acute toxicities. All statistical tests were performed using SPSS ver. 21.0 (IBM, Armonk, NY, USA).

\section{Results}

\section{Patient characteristics}

The characteristics of the 95 patients are summarized in Table 1. The median follow-up time was 21 months (range, 1 to 102 months). Fifteen patients (16\%) had invasive confirmation of T4 status, such as thoracotomy. The most common reason for T4 status was pulmonary artery or vein invasion in 30 patients (32\%) (Table 2).

\section{Treatment characteristics}

The majority of patients ( $n=68,72 \%$ ) received a total RT dose of 66 Gy in 30 fractions; the EQD2 (equivalent dose in 2 Gy fractions) was $67.1 \mathrm{~Gy}(\alpha / \beta 10 \mathrm{~Gy}$ for tumor). The median radiation dose was also EQD2 67.1 Gy (range, 56.9 to $83.3 \mathrm{~Gy}$ ). Five patients were irradiated with $66 \mathrm{~Gy}$, and four patients received $60 \mathrm{~Gy}$ with a fraction size of $2 \mathrm{~Gy}$. The remaining patients received variable doses of 50-77 Gy in fraction sizes of 1.8-10 Gy. One patient was treated with hypofractionated RT using the Arc technique with a dose regimen of $50 \mathrm{~Gy} / 5$ fractions, EQD2 83.3 Gy. This patient had only spine invasion without regional node metastasis.

IMRT was applied to nine patients (9\%) (Table 3). Of these, eight patients had vertebral invasion, and IMRT was used to save their spinal cord. The remaining patient did not have 
vertebral invasion but had a primary lung lesion widely abutted to the thoracic spines, so he also received IMRT to adjust the spinal cord dose. A 64-year-old man diagnosed with cT4NO was treated with hypo-fractionated RT with a fraction size of $10 \mathrm{~Gy}$ up to $50 \mathrm{~Gy}$, and another patient was treated with

Table 1. Patients' characteristics

\begin{tabular}{lc}
\hline \multicolumn{1}{c}{ Characteristic } & No. (\%) \\
\hline Age (yr), median (range) & $64(34-90)$ \\
Gender & \\
Male & $87(92)$ \\
Female & $8(8)$ \\
ECOG performance status & \\
0 & $11(12)$ \\
1 & $73(77)$ \\
2 & $9(9)$ \\
3 & $2(2)$ \\
Weight loss & \\
High ( $\geq 5 \%)$ & $40(42)$ \\
Low (<5\%) & $55(58)$ \\
TNM stage & \\
cT4NOM0 & $57(60)$ \\
CT4N1M0 & $38(40)$ \\
Histology & \\
Squamous cell carcinoma & $67(71)$ \\
Adenocarcinoma & $17(18)$ \\
Large cell carcinoma & $4(4)$ \\
Unspecified & $7(7)$ \\
Chemotherapy & \\
None & $24(25)$ \\
Sequential & $3(3)$ \\
Induction chemotherapy + concurrent & $13(14)$ \\
Concurrent & $55(58)$ \\
\hline
\end{tabular}

ECOG, Eastern Cooperative Oncology Group.

Table 2. Reasons for $T 4^{\mathrm{a}}$

\begin{tabular}{lc}
\multicolumn{1}{c}{ Involvement } & No. (\%) \\
\hline Great vessels & $51(54)$ \\
Pulmonary artery/vein & $30(32)$ \\
Aorta & $16(17)$ \\
Subclavian artery/brachiocephalic vein & $5(5)$ \\
Superior vena cava & $3(3)$ \\
Mediastinum & $27(28)$ \\
Carina/trachea & $13(14)$ \\
Spine & $9(9)$ \\
Heart & $7(7)$ \\
Nerves & \\
Recurrent laryngeal nerve & $5(5)$ \\
Vagus nerve & $1(1)$ \\
Esophagus & $1(1)$ \\
\hline
\end{tabular}

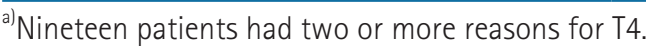

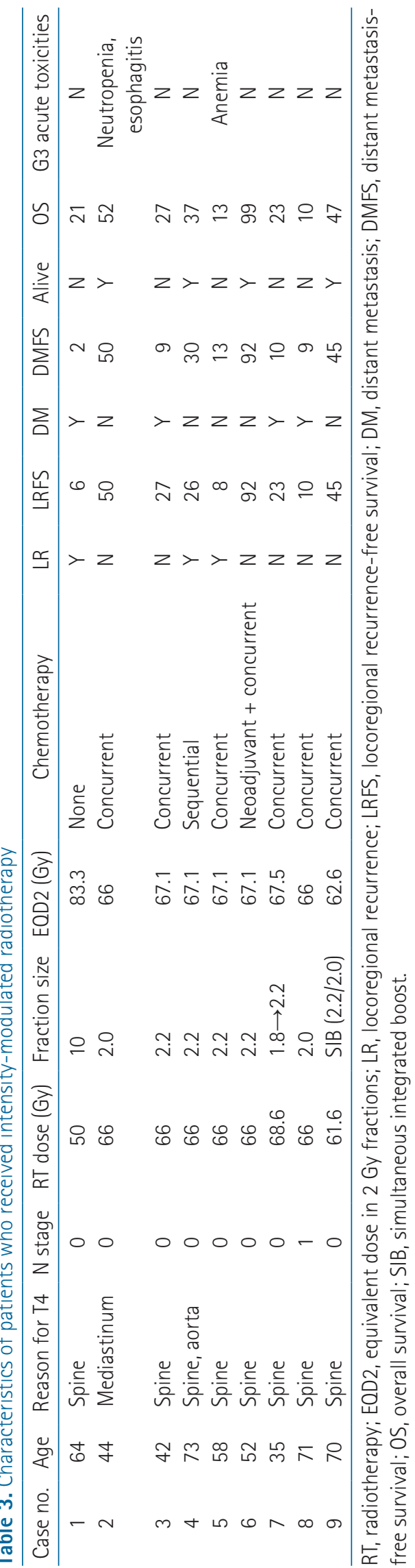

www.e-roj.org 
simultaneous integrated boost. The PTV GTV received $61.6 \mathrm{~Gy}$ in 28 fractions, while the PTV ${ }_{\text {crV }}$ received 56 Gy. LR occurred in three patients (crude rate, 30\%), and four patients experienced DM (crude rate, 44.4\%). OS ranged from 10 to 99 months, and G3 acute toxicities occurred in two patients with neutropenia, esophagitis and anemia. Hematologic toxicities were more likely with concurrent chemotherapy, and the patient with esophagitis initially had a lesion mostly closed to esophagus.

The median RT duration was 44 days (range, 14 to 103 days). Three patients had over 90 days of RT. Initially, the aim of CRT in these patients was induction for surgical resection. However, they remained inoperable despite preoperative CRT and decided to receive additional RT to a definitive dose. There were some extended time intervals in three patients to assess operability.

A total of 71 patients received chemotherapy; 55 patients (78\%, 55/71 patients) were treated with concurrent CRT alone while 13 patients (18\%, 13/71 patients) also received induction chemotherapy. The remaining patients received sequential chemotherapy (4\%, 3/71 patients). The most common CRT regimen was $50 \mathrm{mg} / \mathrm{m}^{2}$ weekly paclitaxel combined with 20 $\mathrm{mg} / \mathrm{m}^{2}$ cisplatin $(\mathrm{n}=48,71 \%$ ). Carboplatin (AUC 2) replaced cisplatin in 14 patients (15\%). The remaining patients received cisplatin combined with gemcitabine $(n=3)$ and etoposide ( $n$ $=3$ ).

The most common regimen in induction/sequential chemotherapy was $1,000 \mathrm{mg} / \mathrm{m}^{2}$ gemcitabine (days 1 and 8 ) with $70 \mathrm{mg} / \mathrm{m}^{2}$ cisplatin (day 1) $(\mathrm{n}=13,81 \%)$ although the number of cycles varied (range, 2 to 4 cycles). Twenty-five

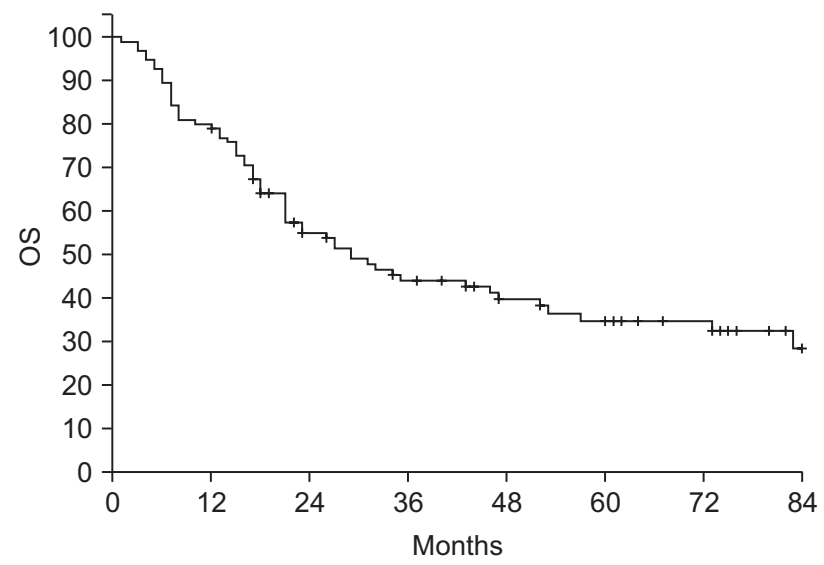

Fig. 1. Overall survival (OS) of 95 T4N0-1MO patients (KaplanMeier).

Table 4. Univariate and multivariate analysis for OS

\begin{tabular}{|c|c|c|c|c|c|c|c|c|}
\hline \multirow{2}{*}{ Variable } & \multirow{2}{*}{ No. } & \multicolumn{4}{|c|}{ Univariate } & \multicolumn{3}{|c|}{ Multivariate } \\
\hline & & Median OS (mo) & SD & $95 \% \mathrm{Cl}$ & $p$-value & $H R$ & $95 \% \mathrm{Cl}$ & $p$-value \\
\hline Age (yr) & & & & & 0.868 & & & - \\
\hline$<60$ & 30 & 27 & 5.91 & $15.42-38.58$ & & - & - & \\
\hline$\geq 60$ & 65 & 31 & 10.83 & $9.78-52.22$ & & & & \\
\hline ECOG performance status & & & & & 0.059 & & & - \\
\hline 0 & 11 & 83 & 17.79 & $148.12-117.88$ & & - & - & \\
\hline 1 & 73 & 27 & 5.52 & $16.19-37.81$ & & & & \\
\hline 2 & 9 & 29 & 15.17 & $0.00-58.73$ & & & & \\
\hline 3 & 2 & 3 & - & - & & & & \\
\hline Weight loss & & & & & 0.000 & & & 0.034 \\
\hline$\geq 5 \%$ & 40 & 17 & 2.64 & $11.84-22.17$ & & 0.38 & $0.23-0.64$ & \\
\hline$<5 \%$ & 55 & 73 & 9.82 & $53.75-92.25$ & & & & \\
\hline Histology & & & & & 0.788 & & & - \\
\hline Squamous cell carcinoma & 67 & 29 & 5.47 & $18.29-39.71$ & & - & - & \\
\hline Adenocarcinoma & 17 & 47 & 13.05 & $21.43-72.57$ & & & & \\
\hline Large cell carcinoma & 4 & 21 & 12.00 & $0.00-44.52$ & & & & \\
\hline Unspecified & 7 & 27 & 14.40 & $0.00-55.23$ & & & & \\
\hline N stage & & & & & 0.156 & & & - \\
\hline No & 57 & 34 & 10.90 & $12.63-55.37$ & & - & - & \\
\hline N1 & 38 & 23 & 5.19 & $12.83-33.18$ & & & & \\
\hline Chemotherapy & & & & & 0.042 & & & 0.045 \\
\hline Any & 71 & 32 & 13.78 & $5.00-59.00$ & & 1.87 & $1.05-3.35$ & \\
\hline None & 24 & 23 & 5.58 & $12.06-33.95$ & & & & \\
\hline
\end{tabular}

OS, overall survival; SD, standard deviation; $\mathrm{Cl}$, confidence interval; HR, hazard ratio; ECOG, Eastern Cooperative Oncology Group. 
percent of patients ( $n=24$ ) received definitive RT alone due to old age, poor ECOG-PS, and/or poor lung function.

\section{Overall survival and failure patterns}

With a median follow-up time of 21 months (range, 1 to 102 months), the median OS was 23 months (range, 1 to 102 months) and the 3-year OS was 44\% (Fig. 1). Pretreatment weight loss and a combination of chemotherapy modalities were significant factors in OS (Table 4). Other factors, such as age ( $<60$ years vs. $\geq 60$ years), ECOG-PS, histologic type, and nodal stage (N0 vs. N1), did not show significance.

Excluding two patients who were followed up at another hospital, a total of 93 patients were included in the failure pattern analysis. The cumulative incidence of $L R$ is shown in Fig. 2. The 3-year cumulative incidence of $L R$ was $48.8 \%$, and LR incidence reached a plateau after 3 years. The 3 -year cumulative incidence of DM was 36.3\% (Fig. 3). The incidence of DM did not increase after 2 years.

The major failure pattern was $L R(n=39,42 \%)$, especially

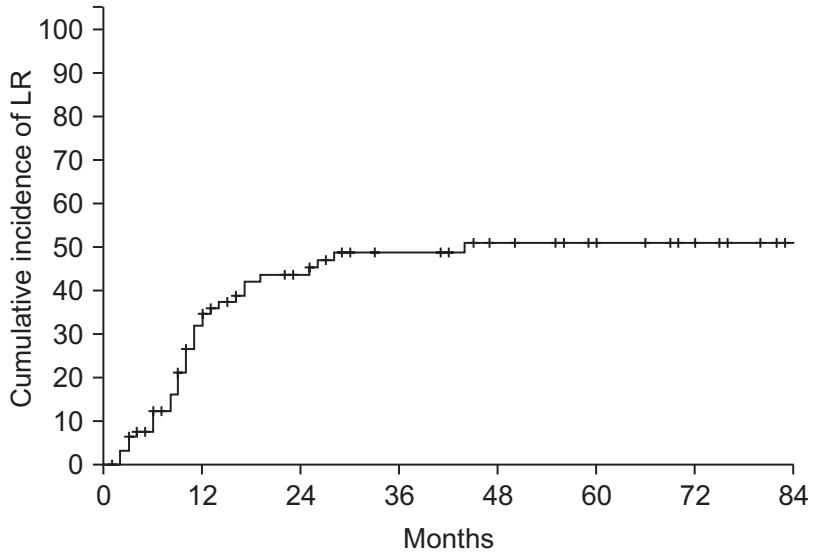

Fig. 2. Cumulative incidence of local recurrence (LR) of 93 T4NO$1 \mathrm{M} 0$ patients (Kaplan-Meier)

Table 5. Patterns of failure $(n=93)$

\begin{tabular}{lc}
\hline & No. of patients (\%) \\
\hline Recurrence & \\
No & $42(45)$ \\
Yes & $51(55)$ \\
Locoregional failure & $39(42)$ \\
Isolated in-field failure & $23(25)$ \\
Isolated out-field nodal failure & $1(1)$ \\
Locoregional failure + distant failure & $15(16)$ \\
Distant failure & $28(29)$ \\
Isolated distant failure & $13(13)$ \\
Locoregional failure + distant failure & $15(16)$ \\
\hline
\end{tabular}

isolated in-field recurrence $(n=23,25 \%)$ (Table 5). One patient experienced isolated out-field recurrence in the contralateral hilar lymph node after 25 months. This patient received salvage RT and remained in disease-free status. DM occurred in 28 patients (29\%), and over half of these patients were also diagnosed with LR $(n=15,16 \%)$.

\section{Toxicities}

Thirteen patients (14\%) had acute toxicities above grade 3 (Table 6). There were 16 total events due to one patient who experienced neutropenia, anemia and thrombocytopenia and another patient with neutropenia and esophagitis. All acute toxicities resolved and did not proceed to chronic complications. Among patients with acute toxicities, 10 received concurrent CRT (with or without induction chemotherapy) and the rest were treated with RT alone. However, there was no statistically significant association between concurrent chemotherapy and acute toxicities.

Grade 3 chronic thrombocytopenia was present in two

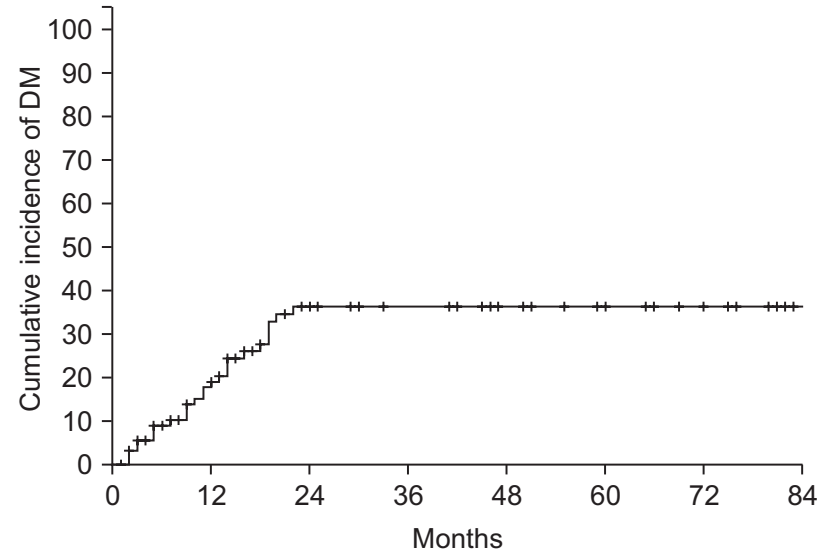

Fig. 3. Cumulative incidence of distant metastasis (DM) of 93 T4N0-1M0 patients (Kaplan-Meier).

Table 6. $\geq$ Grade 3 toxicities

\begin{tabular}{lcccc}
\hline \multirow{2}{*}{ Toxicity } & \multicolumn{2}{c}{ Acute } & & Chronic \\
\cline { 2 - 3 } & CRT & RT alone & p-value & CRT \\
\hline Hematologic & 4 & 1 & & \\
Neutropenia & 5 & 2 & & 0 \\
Anemia & 1 & 0 & & 2 \\
Thrombocytopenia & 3 & 0 & & 1 \\
Esophagitis & 0 & 0 & & 0 \\
Pneumonitis & 13 & 3 & 0.697 & 3 \\
Total events & 10 & 3 & & 3 \\
Total patients & & & & 0 \\
\hline
\end{tabular}

CRT, chemo-radiotherapy; RT, radiotherapy. 
patients, and one patient had grade 3 esophagitis. Two patients received concurrent CRT and one patient was treated with induction chemotherapy followed by concurrent CRT. There were no grade 4 toxicities.

\section{Discussion and Conclusion}

Although the standard treatment for most stage IIIA NSCLC patients is concurrent CRT, there is still controversy over whether surgery has a role in treatment. A prospective study that compared induction concurrent CRT followed by surgery and definitive CRT only included IIIA-N2 disease patients [7]. Although there were no significant difference in 5-year OS in either arms (trimodality $27.2 \%$ vs. bimodality $20.3 \%, p=$ $0.24), 5$-year PFS was significantly improved by trimodality treatment $(22.4 \%$ vs. $11.1 \%, p=0.017)$. After additional matching analysis, the OS was significantly improved in the surgical arm if a lobectomy was performed (36.1\% vs. 17.8\%, $p=0.002)$. However, for pneumonectomy, there was a trend towards poorer OS in the surgical arm (21.9\% vs. $23.6 \%, p=$ not significant).

Although stage IIIA-N2 and T4NO-1 NSCLC patients have similar prognoses, they have different clinical characteristics. T4N0-1 patients have extensive local tumor burden, while N2 disease has less primary tumor burden, with invasion of mediastinal lymph nodes. We expect that T4NO-1 disease would be more difficult to resect than stage IIIA-N2 disease, although there is no definite evidence for this. According to Garrido et al. [11], 22.7\% of T4N0-1 patients and 10.9\% of IIIA-N2 patients were unresectable, even with induction chemotherapy. These patients therefore had a delay in making other treatment choices. The three cases of stage T4NO-1 in the present study had treatment periods above 90 days. Prolonged RT periods lead to concerns about reduced survival.

Achievement of complete resection is an important factor for survival. When patients were treated with surgery alone, the complete resection rates were $37.5 \%-46.2 \%$ and could be improved to $65.9 \%$ with induction chemotherapy $[11,12]$. However, approximately $30 \%$ of patients still needed adjuvant treatment. Despite the effectiveness of adjuvant RT in incompletely resected NSCLC patients, it can be a significant burden [13]. Moreover, surgical resection can cause considerable treatment-related mortality. Albain et al. [7] reported that $7.9 \%$ of NSCLC patients $(n=16)$ died of surgeryrelated causes, while 2.1\% $(n=4)$ in definitive CRT arm died of treatment related causes. Some surgical series have a reported mortality of up to $19.7 \%$ [14]. For this reason, we assumed that definitive CRT would be a better choice for T4NO-1 patients.

Only one study, Reymen et al. [8] has evaluated the treatment outcomes of definitive CRT in T4NO-1 patients, although there are substantial surgical data for this disease $[10,11,14]$ (Table 7). Reymen et al. [8] reported on the longterm survival of stage T4NO-1 and single station IIIA-N2 NSCLC patients treated with definitive CRT. In 42 T4NO-1 patients in that study followed up at a median of 48 months, the median OS was 34 months, with a 3 -year OS of $44 \%$ and a 5 -year OS of 25\%, similar to our present study (median OS 23 months, 3-year OS 44\%, 5-year OS 36.3\%). Compared to previous surgical reports, definitive CRT showed comparable results with complete resection. Completely resected T4NO-1 patients showed a 3-year OS of 41.9\%-71.4\% and a 5-year OS of 27.9\%-53.2\%. Although the survival outcomes were similar, definitive CRT was more feasible when considering treatmentrelated mortality. Postoperative mortality was reported as 0\%-19.1\%, while definitive CRT rarely led to treatment-related death (Table 7).

LR (42\%) was the major failure pattern in our current stage T4NO-1 patients. Isolated in-field recurrence was especially dominant (25\%). This may due to the large volume of primary T4 tumors. There were few reports on failure patterns in T4NO1 patients treated surgically. One previous study reported that the main cause of late death was LR in $58.62 \%$ of patients (17/29 patients). Recurrent tumors were located in the hilar and mediastinal lymph nodes in that study, and in two patients the recurrent tumor was in the left atrium. It seemed that surgery had a similar rate of LR rate [10]. Despite the omission of elective nodal irradiation, out-field nodal recurrence was rare. Distant recurrence occurred in $29 \%$ of patients, and over half of these cases also had LR.

Toxicity was acceptable with 13 events of grade 3 hematologic toxicities in 10 patients $(11 \%)$ and three cases of grade 3 acute esophagitis (3\%). The incidence of grade 3 esophagitis was lower than in a previous study (17\%) [8]. This discrepancy can be attributed to our inclusion of patients receiving definitive RT alone. Also, T4NO-1 patients have a reduced possibility of having their esophagus in the radiation field compared with N2 patients unless the primary mass largely abuts the esophagus. Only one grade 3 chronic esophagitis was reported, and there was no grade 3 radiation pneumonitis. No grade 4 toxicities were reported.

From our present result, we concluded that improvement of in-field local tumor control is the main issue in stage T4NO1 disease. Radiation dose escalation should be considered first. A Radiation Therapy Oncology Group (RTOG) 0617 study 


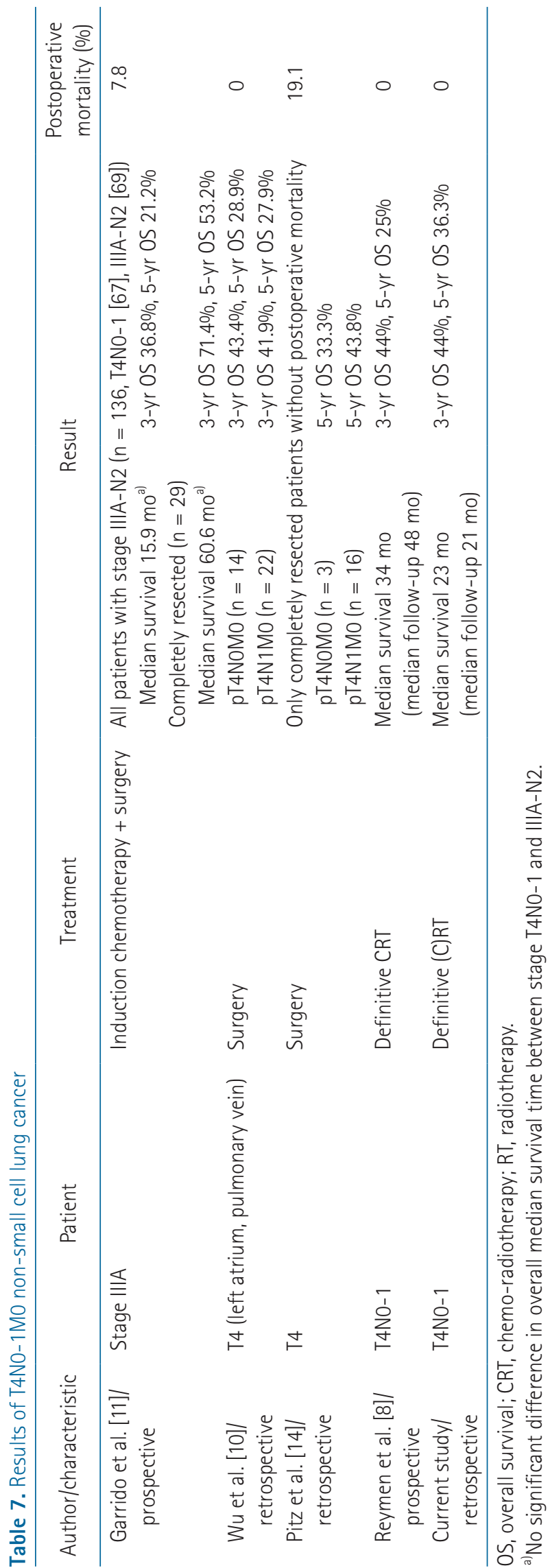

compared standard-dose (60 Gy/30 fractions) and doseescalated (74 Gy/37 fractions) radiation in stage III NSCLC. The outcome was poorer in the dose-escalated arm (median survival 28.7 months vs. 19.5 months, $p=0.0007$ ) without significant differences in toxicities over grade 3 [15]. This result was not clearly explained, and uncertainty remains regarding the intermediate dose escalation between $60 \mathrm{~Gy}$ and $74 \mathrm{~Gy}$. Although many radiation oncologists prescribe intermediate dose levels, intermediate dose escalation was not effective in a previous multi-institutional propensity score matched analysis of stage III NSCLC patients [16]. The RTOG 0617 study enrolled all stage III NSCLC patients and not a specific subgroup. In that study, DM was slightly dominant in both treatment arms (standard dose arm 1.5-year DM, 35.3\% vs. 44\%; high dose arm, $42.4 \%$ vs. $47.8 \%$ ). Mediastinal lymph nodal and distant recurrence might have increased and offset the effect of dose escalation due to the inclusion of patients who had high tumor burden in the nodal area. Our present study focused on T4N0-1 patients who were expected to have less DM. Our center used an intermediate dose escalation regimen of 66 Gy/30 fractions, and the 3-year cumulative LR was $48.4 \%$. We suggest that further dose escalation would improve local tumor control for T4N0-1 patients but not for other stage III subgroups. The next dose escalation phase 3 trials should focus on the T4NO-1 subgroup.

Adoption of IMRT could have safety benefits [17]. In the current study, we did not use IMRT for dose escalation but to satisfy dose constraints. With IMRT, nine patients who could not meet the dose constraints were able to receive the same radiation dose as the other patients. These patients achieved the 3-year OS of $44.4 \%$. The 3-year cumulative incidence of LR was $37.8 \%$ and the 3 -year cumulative incidence of DM was $44.4 \%$. The oncologic outcomes were comparable to those of whole T4N0-1 patients in current study (3-year OS 44\%, 3-year cumulative incidences of LR 48.8\%, 3-year cumulative incidences of DM 36.3\%). They also showed a low rate of toxicities (Table 3). Without dose escalation, IMRT does not seem to affect the oncologic outcomes. We expect better oncologic outcomes with tolerable complication rates through the application of IMRT to whole T4N0-1 patients.

Application of radio-sensitizing agents could also be beneficial. Traditional platinum-based chemotherapy agents are known to have radiation-sensitizing effect [18]. Gemcitabine and paclitaxel were introduced more recently and are thought to be superior radiation sensitizers [19]. Still, there are continuing efforts to find better radio-sensitizing agents. Choy et al. [20] reported the feasibility of pemetrexed. 
Another novel class of radio-sensitizers is nanoparticles (NP). NPs can encapsulate radiation sensitizing agents, protecting them from degradation, or sensitize cancer cells to radiation via physicochemical properties such as high Z number [21].

Combination thereby with surgery should be selectively considered for patients with minimal invasion to adjacent organs expected to obtain complete resection. A multidisciplinary team approach would be appropriate for the decision-making process.

Our present study had some limitations of note. First, staging was mainly based on imaging. Only 16\% of our patients (n = 15) had invasive confirmations such as thoracotomy for T4 staging. PET-CT was routinely checked, so we expected $>-90 \%$ accuracy in the diagnosis of a T4 status; however, some patients might have had T3 tumors [22]. Discriminating $\mathrm{N} 1$ and N2 lymph nodes conglomerated with the primary mass was also difficult. The sensitivity and specificity of CT for detecting mediastinal nodal metastases have been reported as 52\%-64\% and 62\%-69\%, respectively [23]. Therefore, there is a significant risk of understaging nodal status.

Second, patients did not receive the same treatment regimen. Although the majority received definitive CRT, 25\% were treated with RT alone, 15\% received induction chemotherapy, and $2 \%$ received sequential treatment. However, all patients had an appropriate reason for choosing their treatment regimen. The RT-alone group contained patients with old age, poor performance, or poor pulmonary function. Induction chemotherapy was recommended for patients with very large tumors to avoid radiation toxicity. We feel that the present study reflects the real situation in the clinic.

Lastly, this was a retrospective study with inherent biases that could have affected our results.

In conclusion, definitive radiotherapy for T4NO-1 NSCLC results in favorable survival with acceptable toxicity rates. $L R$, especially in-field recurrence, is a major recurrence pattern. For improving local tumor control, the application of IMRT and additional radio-sensitizing agents is needed.

\section{Conflict of Interest}

No potential conflict of interest relevant to this article was reported.

\section{Acknowledgments}

This study was supported by a grant (2013-472) from the Asan Institute for Life Sciences, Seoul, Korea and by Basic Science Research Program through the National Research Foundation of Korea (NRF) funded by the Ministry of Education (NRF2013R1A1A2011346).

\section{References}

1. Dillman RO, Seagren SL, Propert KJ, et al. A randomized trial of induction chemotherapy plus high-dose radiation versus radiation alone in stage III non-small-cell lung cancer. N Engl J Med 1990;323:940-5.

2. Sause WT, Scott $C$, Taylor $S$, et al. Radiation Therapy Oncology Group (RTOG) 88-08 and Eastern Cooperative Oncology Group (ECOG) 4588: preliminary results of a phase III trial in regionally advanced, unresectable non-small-cell lung cancer. J Natl Cancer Inst 1995;87:198-205.

3. Curran WJ. Long-term benefit is observed in a phase III comparison of sequential vs concurrent chemo-radiation for patients with unresected stage III NSCLS: RTOG 94-10. In: 2003 Annual Meeting of the American Society of Clinical Oncology; 2003 June 1; Chicago, IL, USA. Alexandria, VA: ASCO; 2003.

4. Furuse $K_{1}$ Fukuoka $M$, Kawahara $M$, et al. Phase III study of concurrent versus sequential thoracic radiotherapy in combination with mitomycin, vindesine, and cisplatin in unresectable stage III non-small-cell lung cancer. J Clin Oncol 1999;17:2692-9.

5. Rosell R, Gomez-Codina J, Camps C, et al. A randomized trial comparing preoperative chemotherapy plus surgery with surgery alone in patients with non-small-cell lung cancer. N Engl J Med 1994;330:153-8.

6. Albain KS, Rusch VW, Crowley JJ, et al. Concurrent cisplatin/ etoposide plus chest radiotherapy followed by surgery for stages IIIA (N2) and IIIB non-small-cell lung cancer: mature results of Southwest Oncology Group phase II study 8805. J Clin Oncol 1995;13:1880-92.

7. Albain KS, Swann RS, Rusch VW, et al. Radiotherapy plus chemotherapy with or without surgical resection for stage III non-small-cell lung cancer: a phase III randomised controlled trial. Lancet 2009;374:379-86.

8. Reymen $B$, van Baardwijk $A$, Wanders $R$, et al. Long-term survival of stage T4NO-1 and single station IIIA-N2 NSCLC patients treated with definitive chemo-radiotherapy using individualised isotoxic accelerated radiotherapy (INDAR). Radiother Oncol 2014;110:482-7.

9. Ramnath N, Dilling TJ, Harris $\sqcup$, et al. Treatment of stage III non-small cell lung cancer: diagnosis and management of lung cancer, 3rd ed: American College of Chest Physicians evidence-based clinical practice guidelines. Chest 2013;143(5 Suppl):e314S-40S.

10. Wu L, Xu Z, Zhao $X$, et al. Surgical treatment of lung cancer invading the left atrium or base of the pulmonary vein. World J Surg 2009;33:492-6. 
11. Garrido $P$, Gonzalez-Larriba JL, Insa $A$, et al. Long-term survival associated with complete resection after induction chemotherapy in stage IIIA (N2) and IIIB (T4N0-1) non small-cell lung cancer patients: the Spanish Lung Cancer Group Trial 9901. J Clin Oncol 2007;25:4736-42.

12. Chambers $A$, Routledge $T$, Bille $A$, Scarci M. Does surgery have a role in T4N0 and T4N1 lung cancer? Interact Cardiovasc Thorac Surg 2010;11:473-9.

13. Park J, Song SY, Kim SS, et al. Postoperative radiation therapy following the incomplete resection of a non-small cell lung cancer. Radiat Oncol J 2014;32:70-6.

14. Pitz CC, Brutel de la Riviere A, van Swieten HA, Westermann CJ, Lammers JW, van den Bosch JM. Results of surgical treatment of T4 non-small cell lung cancer. Eur J Cardiothorac Surg 2003; 24:1013-8.

15. Bradley JD. A randomized phase III comparison of standard-dose (60 Gy) versus high-dose (74 Gy) conformal chemoradiotherapy with or without cetuximab for stage III non-small cell lung cancer: results on radiation dose in RTOG 0617. In: 49th Annual Meeting of the American Society of Clinical Oncology; 2013 June 7; Chicago, IL, USA. Alexandria, VA: ASCO; 2013.

16. Rodrigues $G$, Oberije $C$, Senan $S$, et al. Is intermediate radiation dose escalation with concurrent chemotherapy for stage III non-small-cell lung cancer beneficial? A multi-institutional propensity score matched analysis. Int J Radiat Oncol Biol Phys 2015:91:133-9.

17. Yom SS, Liao Z, Liu HH, et al. Initial evaluation of treatmentrelated pneumonitis in advanced-stage non-small-cell lung cancer patients treated with concurrent chemotherapy and intensity-modulated radiotherapy. Int J Radiat Oncol Biol Phys 2007;68:94-102.

18. Ball D, Bishop J, Smith J, et al. A randomised phase III study of accelerated or standard fraction radiotherapy with or without concurrent carboplatin in inoperable non-small cell lung cancer: final report of an Australian multi-centre trial. Radiother Oncol 1999;52:129-36.

19. Vokes EE, Herndon JE 2nd, Crawford J, et al. Randomized phase II study of cisplatin with gemcitabine or paclitaxel or vinorelbine as induction chemotherapy followed by concomitant chemoradiotherapy for stage IIIB non-small-cell lung cancer: cancer and leukemia group B study 9431. J Clin Oncol 2002;20: 4191-8.

20. Choy H, Gerber DE, Bradley JD, et al. Concurrent pemetrexed and radiation therapy in the treatment of patients with inoperable stage III non-small cell lung cancer: a systematic review of completed and ongoing studies. Lung Cancer 2015; 87:232-40.

21. Bergs JW, Wacker MG, Hehlgans $S$, et al. The role of recent nanotechnology in enhancing the efficacy of radiation therapy. Biochim Biophys Acta 2015;1856:130-43.

22. Pfannenberg AC, Aschoff $P$, Brechtel $K$, et al. Low dose nonenhanced CT versus standard dose contrast-enhanced $C T$ in combined PET/CT protocols for staging and therapy planning in non-small cell lung cancer. Eur J Nucl Med Mol Imaging 2007;34:36-44.

23. Chheang S, Brown K. Lung cancer staging: clinical and radiologic perspectives. Semin Intervent Radiol 2013;30:99113. 